\title{
Palmoplantar Lichen Planus: A Rare Presentation of a Common Disease
}

\author{
Megan Landis ${ }^{1}$, Christopher Bohyer ${ }^{1}$, Soon Bahrami, 2, Beth Brogan ${ }^{1}$ \\ 1. Department of Dermatology, Indiana University School of Medicine, Indianapolis, IN, USA \\ 2. Department of Pathology, Indiana University School of Medicine, Indianapolis, IN, USA
}

\section{Corresponding author: \\ Beth Brogan, M.D. \\ Department of Dermatology \\ Indiana University School of Medicine}

550 North University Boulevard, Suite 3240

Indianapolis, IN 46202

E-mail: bbrogan@iupui.edu

\section{Key words:}

clobetasol, hand dermatoses, foot dermatoses, lichen planus,

pathology

\begin{abstract}
Background: Lichen planus (LP) is a chronic inflammatory skin disease commonly seen by dermatologists.

Main Observations: The current case describes palmoplantar lichen planus, a rare variant of this disease that can easily be misdiagnosed. This case describes a patient that presented with multiple papules on his hands and plantar feet that had previously been diagnosed as tinea manum and psoriasis. A biopsy from the patient, however, was diagnostic of lichen planus. The patient was subsequently treated successfully with topical clobetasol propionate $0.05 \%$ ointment applied twice daily.
\end{abstract}

Conclusions: Palmoplantar lichen planus is a rare disease that can be easily misdiagnosed. This case report emphasizes the role of the biopsy when presented with such patients.

\section{Introduction}

Lichen planus (LP) is an inflammatory skin disease which produces a characteristic polygonal, violaceous papule. Its slightly scaly surface consists of fine white lines called Wickham's striae, and the lesions are highly pruritic. ${ }^{1}$ There are a number of lichen planus varieties including actinic, annular, atrophic, bullous, hypertrophic, follicular, linear, nail, oral, and ulcerative LP. Another type of LP that has been much less frequently described is palmoplantar LP. We present a case of a palmoplantar LP that had been diagnosed as tinea manum and psoriasis for over five years prior to presentation to our outpatient clinic.

\section{Case Report}

A 59 year-old man presented with a 5 year history of pruritic papules that were initially present exclusively on his palms and soles. Previous diagnoses of palmopalmar psoriasis and tinea manum were made on clinical appearance. He had been treated with topical ketoconazole $2 \%$ cream, a variety of topical steroids, and calcipotriene without relief. The patient had numerous comorbid conditions, including poorly-controlled diabetes mellitus, hypertension, an unspecified seizure disorder, chronic obstructive pulmonary disease, depression, and a history of alcohol abuse. His physical exam on presentation showed multiple $2-3 \mathrm{~mm}$ keratotic papules, some with central dells (Figure 1). A number of these papules had coalesced into plaques, especially on the lateral aspects of the hands. The dorsi of the hands were not involved. The plantar aspects of the feet showed yellow papules and plaques with keratotic scale. More proximal lesions on the ankles and wrists revealed violaceous to brown papules. Some of these had also coalesced into plaques starting at the heels and extending mid-way up the lower legs. The remainder of his exam showed no similar lesions on the trunk or genitalia. His face, nails, and oral mucosa were also spared. The patient complained of intermittent but severe pruritus that compelled him to seek 
therapy. Biopsy taken from the left wrist demonstrated histopathology diagnostic of lichen planus (Figures $2 \mathrm{a}$ and $2 b)$.

The patient was given clobetasol propionate $0.05 \%$ ointment to apply twice daily. Given the reported past failures with topical steroids, acetretin was also considered. Because of his poorly controlled diabetes, we chose not to treat with systemic steroids. The patient's pruritus improved with clobetasol, and his lesions resolved within 4 weeks of initiating therapy. The therapy was well tolerated and without side effects. The patient was subsequently lost to follow-up.

\section{Discussion}

Classic lichen planus (LP) occurs most commonly between the ages of 30 and $60 .{ }^{1}$ It characteristically shows no sex or racial biases, ${ }^{2}$ and the incidence has been reported to represent between $0.14 \%$ to $1.2 \%$ of all dermatologic conditions seen by dermatologists. ${ }^{1}$ Palmoplantar LP, a rare variant of this common disease, parallels the demographic trends of the classic variant, with the exception that the palmoplantar variant is more commonly seen in men than women. ${ }^{3}$

The papules of classic LP preferentially involve the flexural sites, and involvement of the wrist is particularly characteristic. The arms and legs are the most common sites of involvement, although the thighs, lower back, trunk, and neck may also bear lesions. The face and scalp, as well as the palms and soles, are usually spared in classic LP. ${ }^{2}$ Palmoplantar LP is more frequently on the soles than the palms, with the internal plantar arch being the most common site of occurrence. ${ }^{3}$

Lichen planus may develop rapidly over several days into an acute generalized dermatitis. The papules may be scattered or may become grouped into larger lesions, and Koebner phenomenon is commonly observed. Mucus membranes are affected in more than one half of patients, most commonly the buccal mucosa and the tongue. The mucosal lesions are lacy, reticulated white streaks, white papules and plaques, and atrophic and ulcerated patches. ${ }^{1}$

Palmoplantar lesions, in contrast to classic LP lesions, do not have Wickham's striae present and are not shiny. The differential diagnosis includes acquired palmoplantar keratoderma, psoriasis, verruca vulgaris, callus, xanthomas, syphilis, Kyrle disease, acrokeratosis paraneoplastica, punctate porokeratosis, lichen simplex chronicus, 4 tinea pedis/tinea manum, and eczematous hand dermatitis. Since the clinical features in palmoplantar LP are not always suggestive of the diagnosis, as demonstrated by our patient, biopsy is extremely useful.

The histopathology of LP is diagnostic and generally consistent across different clinical variants, including the palmoplantar variant. It consists of hyperkeratosis with focal wedge-shaped hypergranulosis, irregular acanthosis, liquefaction degeneration of the basal cell layer, and a

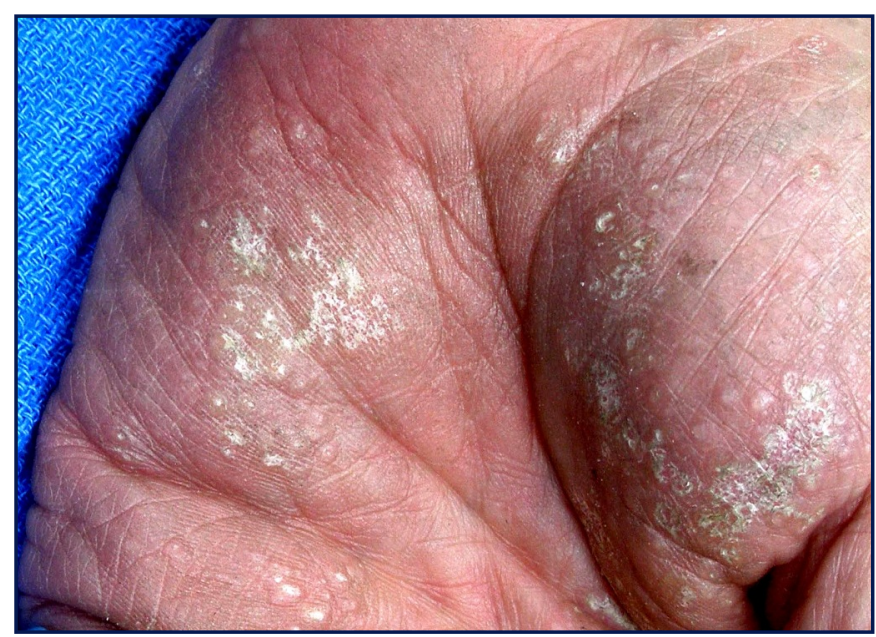

Figure 1

The patient's palmar surface reveals multiple 2-3 mm keratotic papules, some with central dells.

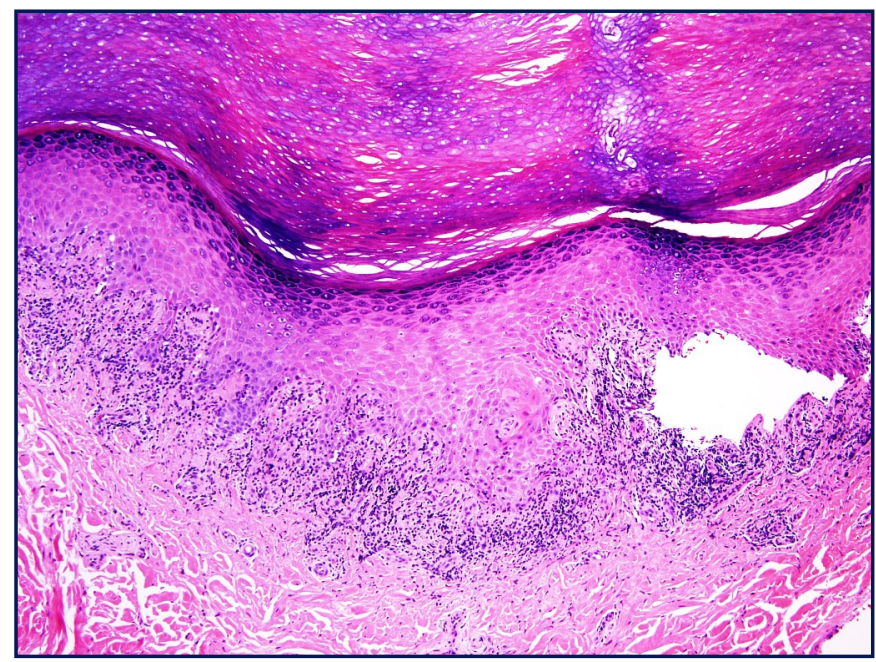

Figure $2 \mathrm{~A}$ and $2 \mathrm{~B}$

A biopsy shows typical features of lichen planus; $(A)$ note wedge shaped hypergranulosis, saw-toothing of rete pegs, and interface changes. A Max-Joseph space is present at the right. (H\&E, 100X), (B) At high power, basal vacuolar change and colloid bodies are identified at the dermal-epidermal junction. (H\&E, 400X).

bandlike superficial dermal lymphohistiocytic infiltrate. ${ }^{5}$ Colloid bodies, also called hyaline, cytoid, or Civatte bodies, are commonly present in the deep epidermis and superficial dermis. Small separations between the epidermis and the dermis may be present and are called Max Joseph spaces. Saw-toothing of the papillae is another commonly seen histopatholgic feature of LP. ${ }^{6}$

LP has been described in the literature with morphological patterns including erythematous scaly (most common), hyperkeratotic, keratoderma-like, punctate, and ulcerative. ${ }^{4}$ Palmoplantar LP has been further characterized by the presence of pruritic erythematous scaly and/or hyperkeratotic plaques, with well-defined edges, located on the internal plantar arch, without involvement of the fingertips, and which usually disappears within a few months. ${ }^{3}$ 
The largest to date case series of palmoplantar LP described 36 patients who also had lesions outside the palms and soles. ${ }^{3}$ Nine patients presented with lesions classified as hyperkeratotic, consisting of firm, yellow papules and plaques ranging from $0.5 \mathrm{~cm}$ in diameter to large plaques encompassing the palms and soles similar to those present in our case. The other 27 patients exhibited an erythematous scaly pattern, with erythematous, scaly plaques ranging in size from $0.5 \mathrm{~cm}$ to $10 \mathrm{~cm}$. The lesions were mostly bilateral, pruritic, and commonly located on the internal plantar arch, the central area of the palm, and the thenar and hypothenar eminences. The histopathologic features of palmoplantar LP are as described for classic LP. Parakeratosis, however, which is not usually a feature of classic LP, was described in over half of the biopsies in this case series. Patients responded to topical and/or oral treatment with steroids and antihistamines. Authors do not provide information about the type or dose of steroids. Thirty-two patients were monitored during their respective treatment durations, which ranged from 21 days to 8 months. Patients who received oral corticosteroids clinically improved between the first and third weeks of treatment, with total clearance after a mean of 2.9 months. The authors did not indicate the names or doses of the medications. Twenty-nine percent of patients had recurrences, none of which recurred on palmoplantar surfaces.

In addition to treatments used for the classic LP variant, including topical and oral steroids, other treatments unique to the palmoplantar variant have included surgical excision with split-thickness skin grafting. ${ }^{6,7,8}$ Moss and Harman described case reports of skin grafts of both palmar and plantar lichen planus following failure of medical treatment. 6 They found that the skin grafts were not subsequently involved in the disease process and they withstood the demands of being on a weight-bearing surface, confirming an earlier report by Cram et al. ${ }^{9}$ Alternatively, De Jong and Kerkhof described a case of lichen planus/lupus erythematosus overlap syndrome with involvement of both the palms and soles that was successfully treated with acitretin. 10 The dose of acitretin was started at 35 $\mathrm{mg}$ daily with a subsequent increase to $50 \mathrm{mg}$ daily. Acitretin was continued until clearance at 12 weeks, at which time the dose was gradually tapered. Mild chelitis was the only noted side effect.

Another recently described case of biopsy-proven palmoplantar LP that was resistant to emollients and topical corticosteroids, was successfully treated with a course of cyclosporin. ${ }^{11}$ The initial cyclosporin dose of 3.5 $\mathrm{mg} / \mathrm{kg} /$ day was continued for 4 weeks, at which time visible clinical improvement was observed. The dose was tapered over the following 4 weeks. The complete 8 week course of cyclosporin was therapeutically effective and well-tolerated with no significant side effects. the skin and mucus membranes. We describe a case of a rare variant of LP, palmoplantar LP, to remind readers of this abnormal presentation that can be mistaken for other more common palmar inflammatory conditions. Since clinical features may not be suggestive of LP, biopsy is vital to establish diagnosis.

\section{References}

1. Fox BJ, Odom RB. Papulosquamous diseases: a review. J Am Acad Dermatol. 1985; 12:597-624.

2. Boyd AS, Neldner KH. Lichen Planus. J Am Acad Dermatol. 1991; 25:593-619.

3. Sanchez-Perez J, Rios Buceta L, Fraga J, Garcia-Diez A. Lichen planus with lesions on the palms and/or soles: prevalence and clinicopathological study of 36 patients. $\mathrm{Br} J$ Dermatol. 2000; 142:310-314.

4. Rotunda AM, Craft N, Haley JC. Hyperkeratotic plaques on the palms and soles. Arch Dermatol. 2004; 140:1275-1280.

5. Zaias N. The nail in lichen planus. Arch Dermatol. 1970; 101:264-271.

6. Moss ALH, Harman RRM. Surgical treatment of painful lichen planus of the hand and foot. Br J Plast Surg. 1986; 39:402-407.

7. King D, Karkowski J, Miller SH. Plantar lichen planus - treatment by exicison and skin grafting. Plast Recon Surg. 1975; 56:668-670.

8. Lendrum, J. Surgical treatment of lichen planus of the soles. Br J Plast Surg. 1974; 27:171-175.

9. Cram DL, Kierland RR, Winkelman RK. Ulcerative lichen planus of the feet. Arch Dermatol. 1966; 93:692-701.

10. De Jong EM, Van De Kerkhof PC. Coexistance of palmoplantar lichen planus and lupus erythematosus with response to treatment using acitretin. Br J Dermatol. 1996; 134:538-541.

11. Karakatsanis G, Patsatsi A, Kastoridou C, Sotiriadis D. Palmoplantar lichen planus with umbilicated papules: an atypical case with rapid therapeutic response to cyclosporin. J Eur Acad Dermatol Venereol. 2007; 21:1006-1007.

\section{Conclusion}

Lichen planus is an inflammatory dermatitis found on 